\title{
Are ready-to-eat salads ready to eat? An outbreak of Salmonella Coeln linked to imported, mixed, pre-washed and bagged salad, Norway, November 2013
}

\author{
D. F. VESTRHEIM ${ }^{1,2} *$ H. LANGE ${ }^{1,3}, \mathrm{~K} . \mathrm{NYGARD}^{1}, \mathrm{~K} . \mathrm{BORGEN}^{1}$, \\ A. L. WESTER ${ }^{1}$, M. L. KVARME ${ }^{4}$ AND L. VOLD ${ }^{1}$ \\ ${ }^{1}$ Division of Infectious Disease Control, Norwegian Institute of Public Health, Oslo, Norway \\ ${ }^{2}$ European Programme for Public Health Microbiology Training (EUPHEM), European Centre for Disease \\ Prevention and Control, (ECDC), Stockholm, Sweden \\ ${ }^{3}$ European Programme for Intervention Epidemiology Training (EPIET), European Centre for Disease \\ Prevention and Control, (ECDC), Stockholm, Sweden \\ ${ }^{4}$ Norwegian Food Safety Authority, Ass, Norway
}

Received 10 June 2015; Final revision 8 October 2015; Accepted 21 October 2015;

first published online 20 November 2015

\section{SUMMARY}

We investigated a nationwide outbreak of Salmonella Coeln in Norway, including 26 cases identified between 20 October 2013 and 4 January 2014. We performed a matched case-control study, environmental investigation and detailed traceback of food purchases to identify the source of the outbreak. In the case-control study, cases were found to be more likely than controls to have consumed a ready-to-eat salad mix (matched odds ratio $20,95 \%$ confidence interval $2 \cdot 7-\infty$ ). By traceback of purchases one brand of ready-to-eat salad was indicated, but all environmental samples were negative for Salmonella. This outbreak underlines that pre-washed and bagged salads carry a risk of infection despite thorough cleaning procedures by the importer. To further reduce the risk of infection by consumption of ready-to-eat salads product quality should be ensured by importers. Outbreaks linked to salads reinforce the importance of implementation of appropriate food safety management systems, including good practices in lettuce production.

Key words: Foodborne infections, outbreaks, Salmonella.

\section{INTRODUCTION}

Salmonella infection is notifiable to the Norwegian Surveillance System for Communicable Diseases (MSIS). Salmonellosis is the second most common acute bacterial enteritis notified in Norway [1]. In 2013, 1364 cases were notified (incidence rate 27.5/ 100000 population). More than $75 \%$ of notified infections are acquired abroad. The most prevalent serovar is Salmonella Enteritidis, followed by Salmonella

\footnotetext{
* Author for correspondence: D. F. Vestrheim, Department of Bacteriology and Immunology, Norwegian Institute of Public Health, PO Box 4404 Nydalen, 0403 Oslo, Norway.

(Email: didrik.frimann.vestrheim@fhi.no)
}

Typhimurium. Other serovars constitute about 50\% of notified cases. Salmonella Coeln is a rare nontyphoid serovar of Salmonella, with up to three cases notified annually to MSIS in the past 10 years. One previous outbreak of $S$. Coeln associated with consumption of minced meat in France in 1998 has been reported [2].

With the exception of $S$. Typhimurium, Salmonella is not endemic in Norway. Domestically acquired salmonellosis caused by other serovars thus implies consumption of contaminated imported food products. Fresh produce, such as herbs and lettuce, is a known vehicle for foodborne pathogens, including Salmonella, and several outbreaks have been described [3-6]. The combination of Salmonella and leafy greens eaten raw has 
been ranked first in a listing of pathogen and food of nonanimal origin combinations linked to human cases [7].

Faecal specimens obtained from cases presenting with gastroenteritis in Norway are routinely tested for Salmonella at diagnostic microbiological laboratories. When identified, Salmonella isolates are forwarded to the National Reference Laboratory for enteric pathogens (NRL) at the Norwegian Institute of Public Health (NIPH) for verification and serotyping. A case is notified by a clinician, the diagnostic laboratory and NRL to MSIS, where notifications are linked in one record using a unique personal identification number.

On 18 November 2013, the NRL alerted the Department of Infectious Disease Epidemiology, NIPH, of a cluster of four isolates of $S$. Coeln submitted to the laboratory in the period 1-18 November 2013. The isolates originated from cases of gastroenteritis residing in different parts of Norway. A nationwide common-source outbreak was suspected. According to national guidelines, investigation of nationwide outbreaks are coordinated by NIPH [8], and thus an outbreak investigation was initiated by NIPH in collaboration with the Food Safety Authority and the Municipal Health Authorities, in order to determine the extent of the outbreak, identify the source and suggest possible preventive measures.

\section{METHODS}

\section{Outbreak investigation}

We defined a case as a person living in Norway with a laboratory-confirmed infection with $S$. Coeln, with onset of symptoms on 20 October 2013 or later, and with no history of travel outside Norway in the 2 weeks preceding onset of symptoms. Isolates were verified as Salmonella enterica spp. enterica by conventional biochemical tests, and identified as $S$. Coeln by serotyping according to the White-Kauffmann-Le Minor scheme [9], and, due to the rare and sporadic occurrence of this serovar in Norway, no characterization by molecular methods was undertaken to confirm relatedness of isolates.

According to the Norwegian Infectious Diseases Control Act a specific ethical clearance is not required for investigation of outbreaks [10].

\section{Epidemiological investigation}

The cases were interviewed face-to-face by local Food Safety Authorities. In order to identify possible common exposures and to generate hypotheses, the first ten cases, notified by 20 November, were interviewed using a standard trawling questionnaire

The next consecutive cases, notified from 21 to 26 November 2013, were included in a matched casecontrol study to test the hypotheses. A structured questionnaire was developed, focused on a limited number of suspected food items identified in the trawling interviews (Table 1). The cases were interviewed about consumption of suspected food items during the week before symptom onset.

Cases notified after 26 November 2013, including suspected secondary cases, were interviewed but not included in the case-control study.

For each of the cases included in the case-control study we recruited three controls. The controls were matched by age, sex and municipality, using the Norwegian population registry and the phone registry. The controls were interviewed over the phone by NIPH staff, using the same structured questionnaire as for cases, focusing on consumption of suspected food items in the 2 weeks preceding the interview.

Questionnaire response data were entered in a webbased questionnaire (Questback, www.questback. com), exported to Excel (Microsoft, USA), and cleaned and analysed in Stata v. 12.1 (StataCorp., USA). We compared each case to three matched controls to estimate the association between gastrointestinal illness and exposure to food items using matched odds ratios (mOR) with 95\% confidence intervals (CI).

\section{Environmental investigation and traceback investigation}

The Food Safety Authority obtained specimens for microbiological testing from suspected food items during home visits to cases. Restaurants visited by cases in the relevant time period were tracked and food samples were requested.

Detailed data on purchases of food items from grocery stores were obtained from four cases. The time points for credit card transactions were retrieved from bank statements, and by using the time points for purchases, detailed information on purchased products were identified by linking to anonymous copies of registered till receipts from grocery stores.

\section{RESULTS}

By 26 November 2013, we had identified a total of 19 cases; ten $(52 \cdot 6 \%)$ were female and the median age was 37 (range 2-81) years. Date of symptom onset 
Table 1. Univariate analysis of matched odds ratios for infection with Salmonella Coeln in the outbreak in Norway, November 2013. In addition to the food items listed in the table, cases and controls were asked specifically about consumption of fresh herbs and sprouts, cheese, rice, porridge, and eggs

\begin{tabular}{llllll}
\hline \hline Exposure & No. of cases $(n=7)$ & No. of controls $(n=24)$ & Matched OR & $95 \%$ CI & $P$ value \\
\hline Ready-to-eat baby leaf mix & 5 & 0 & 20 & $(2 \cdot 7-\infty)$ & $0 \cdot 002$ \\
Baby leaf salad & 2 & 0 & $7 \cdot 2$ & $(0 \cdot 6-\infty)$ & $0 \cdot 125$ \\
Field salad & 2 & 0 & $7 \cdot 2$ & $(0 \cdot 6-\infty)$ & $0 \cdot 125$ \\
Omelette & 2 & 0 & $7 \cdot 2$ & $(0 \cdot 6-\infty)$ & $0 \cdot 125$ \\
Ready-to-eat rucola salad & 3 & 1 & $5 \cdot 6$ & $(0 \cdot 4-310)$ & $0 \cdot 281$ \\
Chicken & 4 & 8 & $2 \cdot 6$ & $(0 \cdot 3-32)$ & $0 \cdot 508$ \\
Iceberg salad & 3 & 8 & $2 \cdot 4$ & $(0 \cdot 3-31)$ & $0 \cdot 625$ \\
\hline \hline
\end{tabular}

OR, Odds ratio; CI, confidence interval.

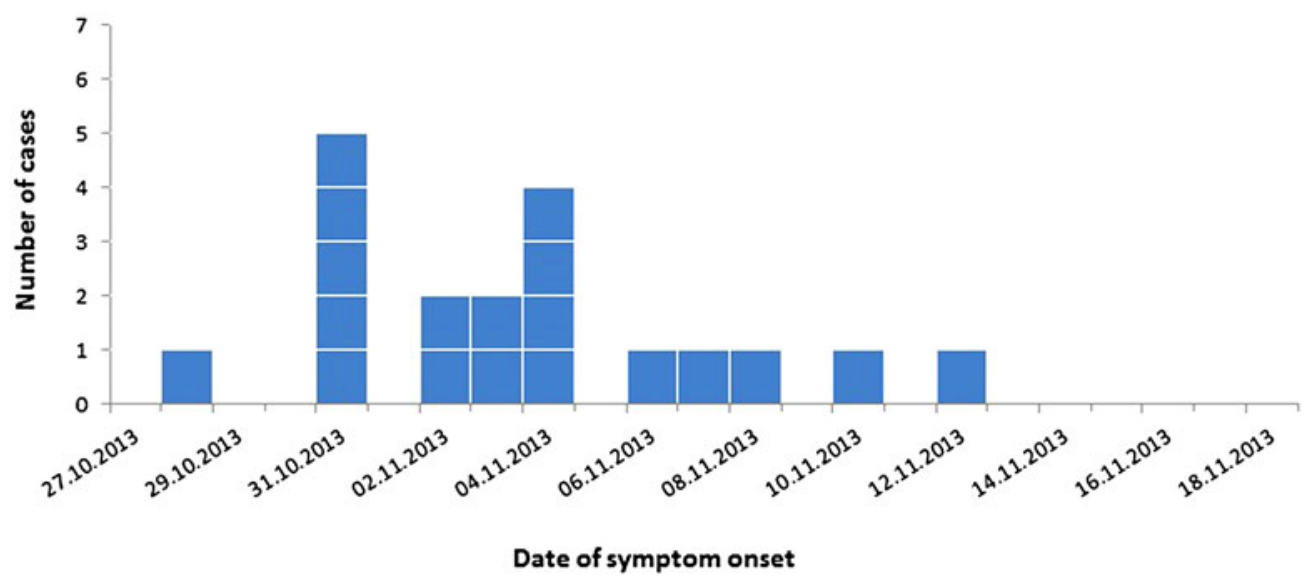

Fig. 1. Cases infected with Salmonella Coeln and notified by 26 November in the outbreak in Norway, 2013, by date of symptom onset.

was between 28 October and 12 November (Fig. 1). The cases were geographically dispersed in both rural and urban areas (Fig. 2).

From December 2013 to January 2014, seven additional cases were reported; four cases reported having a household member or having close contact with an individual with symptoms of diarrhoea preceding disease and were presumed to have a secondary infection; two cases with $S$. Coeln isolated from a urinary tract infection reported preceding gastrointestinal complaints; one primary case with symptom onset on 21 November 2013. These cases were not included in the case-control study.

Of 19 cases notified by 26 November, eight cases were included in the case-control study. One of the cases did not respond, leaving the number of cases in the analysis as seven. We recruited 24 controls. More than $50 \%$ of cases reported consuming chicken and ready-to-eat baby leaf mix. In univariate analysis, we found cases to be more likely than controls to have consumed baby leaf mix (mOR 20, 95\% CI $2 \cdot 7-\infty$ ) (Table 1).

A total of 39 environmental samples were taken; 24 samples from the households of five cases and 15 samples from six restaurants. Of these samples, three were obtained from leftover ready-to-eat salad and salad bags. All the environmental samples were negative for Salmonella.

One specific brand of ready-to-eat salad was singled out by information from till receipts retrieved using detailed information of credit card purchases from two cases. This brand was also mentioned specifically in three case interviews. The supplier of this product used available information to track ingredients and shipments of salad included in the ready-to-eat salad mix. By comparison with the geographical spread of cases, the distribution of different batches of the product within Norway pointed towards one of the imported ingredients in the salad mix. The Norwegian importer and distributor, which was certified according 


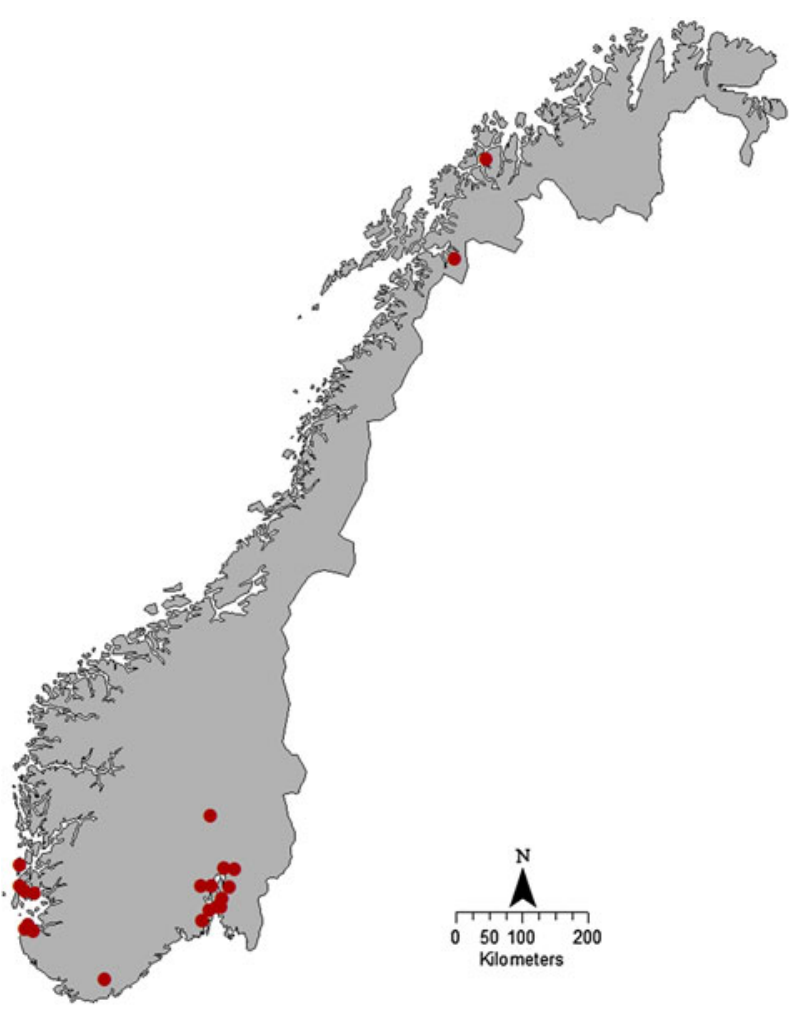

Fig. 2. Spot map showing the geographical distribution of cases of Salmonella Coeln in the outbreak in Norway, 2013. One spot equals one case.

to the British Retail Consortium's Global Standard for Food Safety, inspected the facilities used for washing and packing of salad in Norway, including microbiological sampling of washing water. The production facilities in the country of origin of the salad ingredients were also inspected by the Norwegian importer. No irregularities in the production chain were identified by these inspections and analyses. Import of the suspected salad ingredient was temporarily stopped.

We informed the public of the outbreak and the results of the investigation by postings on the websites of NIPH and the Food Safety Authorities. The shelf-life of pre-washed and bagged salad is short, usually in the range of 8-12 days with a 'best before' date printed on the bag. The production lot with the suspected product had passed the expiry date and was no longer on the market by the end of November, and no further recall of the product was done.

\section{DISCUSSION}

We identified ready-to-eat salad mix as the most likely source of this outbreak of $S$. Coeln in Norway. Mixes of baby leaves consist of small leaf lettuce such as rucola, baby spinach and red rhubarb (a type of chard), which in this case were imported, washed and bagged in Norway. Pre-washed and bagged salad products have a short shelf-life, are marked with a 'best before' date, and each lot is on the market for a limited time period.

In this outbreak we combined analytical epidemiology, environmental investigations and meticulous traceback of suspected food items. We used detailed information from credit card transactions to identify and narrow down suspected items and production lots of ready-to-eat salad. In addition, the distributor visited and inspected producers of suspected salad mix ingredients. Despite these efforts, we were unable to identify $S$. Coeln in the suspected food item.

Outbreaks resembling this have occurred several times in the past, including outbreaks caused by small leaf salads such as rucola [3], and outbreaks caused by ready-to-eat salad products [4, 11, 12]. Contamination can occur at all stages of the farmto-fork continuum, and outbreaks occur despite the emphasis on good management practices in production of fresh produce. The main entry point for contaminants is at pre-harvest level, e.g. due to contaminated irrigation water [13]. Once introduced in the produce, a load reduction of the contaminating pathogen can be attempted by washing procedures. However, post-harvest processing may typically achieve a bacterial load reduction in the range of 1-2 logs of colony-forming units/gram, even in the presence of sanitizers [14, 15]. Industrial high volume processing, including washing and cutting, might also introduce a risk for cross-contamination of the produce, e.g. by re-use of wash water $[14,16]$. Elimination of contamination at post-harvest level may be further hampered by internalization of bacteria into the plant tissue during growth $[16,17]$. Thus, pre-washing performed by importers before distribution might not sufficiently reduce or eliminate the risk of infection, even when conforming to international standards for certification. However, the labelling of ready-to-eat salads clearly states that further washing is unnecessary, and the responsibility for ensuring product safety thus lies with the importers. Although no breaks in processing routines were found in this outbreak, the Norwegian importer and distributor made changes to their procedure for washing of salads, by increasing the volume of water used for washing, and by more frequent quality checks of wash water.

Vigilance for Salmonella outbreaks is high in Norway, and outbreaks might be more easily detected than in countries where salmonellosis is endemic. This outbreak was identified and suspected by the NRL by 
a cluster of more than expected isolates of a rare Salmonella serovar over a short period of time. If the outbreak strain had belonged to a more prevalent serovar that is not routinely subtyped by molecular methods, the outbreak might have passed undetected.

On the other hand, as a consequence of a low baseline risk in Norway, consumers might take less care when preparing food with risk of contamination. Whether additional washing by the consumer would have reduced the risk for infection is doubtful and difficult to control. With increased consumption of fresh fruit and vegetables as part of a recommended healthy diet, outbreaks from contaminated produce will occur. Identification and investigation of gastroenteritis outbreaks provide an opportunity to study flaws in the farm-to-fork production chain and identify areas for improvements.

\section{CONCLUSIONS AND RECOMMENDATIONS}

This outbreak is one of an increasing number of outbreaks linked to ready-to-eat salads. This underlines that enteric infections may still occur through contaminated fresh leafy greens despite apparently thorough cleaning prior to cutting and packing. Hence, emphasis should be put on eliminating microbiological hazards in primary production [18]. Appropriate implementation of food safety management systems including Good Agricultural Practices (GAP), Good Hygiene Practices (GHP) and Good Manufacturing Practices (GMP), should be primary objectives of salad production and processing.

\section{ACKNOWLEDGEMENTS}

We gratefully acknowledge the municipal health authorities and the microbiological laboratories in the affected areas who participated in the outbreak investigation. We thank personnel at the Norwegian Food Safety Authority for conducting interviews with casepatients, collecting food samples, and collaborating on the international investigation.

\section{DECLARATION OF INTEREST}

None.

\section{REFERENCES}

1. Lange $\mathbf{H}$, et al. Annual report. Food- and waterborne infections, 2013. Norwegian Institute of Public Health, 2014.
2. Haeghebaert $\mathbf{S}$, et al. Minced beef and human salmonellosis: review of the investigation of three outbreaks in France. Eurosurveillance 2001; 6: 21-26.

3. Nygard K, et al. Outbreak of Salmonella Thompson infections linked to imported rucola lettuce. Foodborne Pathogens and Disease 2008; 5: 165-173.

4. MacDonald E, et al. Yersinia enterocolitica outbreak associated with ready-to-eat salad mix, Norway, 2011. Emerging Infectious Diseases 2012; 18: 1496 1499.

5. Irvine WN, et al. Investigation of an outbreak of Salmonella enterica serovar Newport infection. Epidemiology and Infection 2009; 137: 1449-1456.

6. Sagoo SK, et al. Microbiological sudy of ready-to-eat salad vegetables from retail establishments uncovers a national outbreak of salmonellosis. Journal of Food Protection 2003; 66: 403-409.

7. Da Silva Felício MT, et al. Risk ranking of pathogens in ready-to-eat unprocessed foods of non-animal origin (FoNAO) in the EU: initial evaluation using outbreak data (2007-2011). International Journal of Food Microbiology 2015; 195: 9-19.

8. Kapperud G, Nygard K. Outbreak Manual, Smittevern, vol. 17, 2009. Norwegian Institute of Public Health, Oslo, Norway.

9. Grimont PAD, Weill F-X. Antigenic formulae of the Salmonella serovars, 2007. Institut Pasteur.

10. Infection Disease Control Act (Smittevernloven). (www. lovdata.no/lov/1994-08-05-55). Accessed 10 June 2015.

11. Launders $\mathbf{N}$, et al. Outbreak of Shiga toxin-producing $E$. coli $\mathrm{O} 157$ associated with consumption of watercress, United Kingdom, August to September 2013. Eurosurveillance 2013; 18. pii $=20624$.

12. Little CL, Gillespie IA. Prepared salads and public health. Journal of Applied Microbiology 2008; 105: 1729-1743.

13. Park S, et al. Risk factors for microbial contamination in fruits and vegetables at the preharvest level: a systematic review. Journal of Food Protection 2012; 75: 20552081.

14. Warriner K, et al. Recent advances in the microbial safety of fresh fruits and vegetables. Advances in Food and Nutrition Research 2009; 57: 155-208.

15. Doyle MP, Erickson MC. Summer meeting 2007 - the problems with fresh produce: an overview. Journal of Applied Microbiology 2008; 105: 317-330.

16. Lynch MF, Tauxe RV, Hedberg CW. The growing burden of foodborne outbreaks due to contaminated fresh produce: risks and opportunities. Epidemiology and Infection 2009; 137: 307-315.

17. Kroupitski Y, et al. Internalization of Salmonella enterica in leaves is induced by light and involves chemotaxis and penetration through open stomata. Applied and Environmental Microbiology 2009; 75: 6076-6086.

18. De Giusti M, et al. The evaluation of the microbial safety of fresh ready-to-eat vegetables produced by different technologies in Italy. Journal of Applied Microbiology 2010; 109: 996-1006. 